\title{
Post-mortem examination of a small intestine in the coeliac syndrome
}

\author{
B. CREAMER AND P. LEPPARD \\ From the Gastro-Intestinal Laboratory, St Thomas's Hospital, London
}

EDITORIAL SYNOPSIS This is an illustrated account of the post-mortem appearances of the small intestine from a patient with a secondary coeliac syndrome. It shows the value of the dissecting microscope in the examination of such material.

Histological examination of the small intestine after death is almost invariably valueless because of autolysis. This is the reason why the lesion of idiopathic steatorrhoea or coeliac syndrome has only been recognized in recent years with the introduction of peroral biopsy. These biopsies can be profitably examined under the dissecting microscope (Rubin, Brandborg, Phelps, and Taylor, 1960; Holmes, Hourihane, and Booth, 1961) but as yet there have been no reports of post-mortem material examined in this way.

This paper describes and illustrates the postmortem changes in the small intestine from a patient who died with a coeliac syndrome secondary to reticulum cell sarcoma (Hindle and Creamer, 1965). The material also shows the difference between the ante-mortem and post-mortem appearances of the intestine as viewed through the dissecting microscope.

\section{METHODS}

The whole small intestine of a man with secondary coeliac syndrome was available for study. Specimens about $2 \mathrm{~cm}$. square were taken every 8 in. from the upper duodenum to the terminal ileum; there were 23 specimens. They have been numbered 1 to 23 from duodenum to ileum. They were pinned flat without obliterating the mucosal folds and stained with $5 \%$ alcoholic eosin and cleared in xylol. Each specimen was examined under the dissecting microscope and several areas photographed, always recording separately appearances on the crests of the mucosal folds and in the troughs between them.

\section{CASE HISTORY}

The patient was a man of 55 who developed diarrhoea, weight loss, oedema, tetany, and a sore tongue and was admitted in September 1963 after an illness of five months. He presented with the typical appearances of malabsorption and had a faecal fat excretion of $26 \cdot 1 \mathrm{~g}$. per day. Investigations showed a megaloblastic anaemia with folic acid deficiency, low serum calcium, hypoproteinaemia, and positive evidence of exudative enteropathy. A small bowel meal showed an abnormal appearance compatible with steatorrhoea and jejunal biopsy showed a flat mucosa.

He was treated on a gluten-free diet and improved remarkably but two weeks after starting this, spontaneously perforated in the mid-ileum. Eight inches of ileum were resected and he made an uninterrupted recovery. Examination of the specimen showed a simple perforation without any tumour mass but histology revealed a small area on the edge of the perforation which looked like reticulum cell sarcoma. Under the dissecting microscope the mucosa showed a convoluted pattern on the crests of the mucosal folds and a leafy appearance in the troughs. Histology confirmed this showing a marked difference between the crests and the troughs. The patient continued to respond to the diet and regained normal health and weight. In April 1964 diarrhoea and wasting reappeared and he was readmitted. This time his appearance was similar to that on the first admission except that he had enlarged glands in an axilla and one groin. Biopsy of these showed reticulum cell sarcoma. He again had a full malabsorption syndrome and a flat jejunal mucosa. He was treated with chlorambucil, 5 mg. t.d.s., and the remaining glands shrank and disappeared in one month. During this time two further jejunal biopsies showed no significant change except for some diminution in mitosis. However, he went steadily downhill and died with gross emaciation. Necropsy was carried out twentyfour hours after death. There was no evidence of lymphoma in any part of the body. The small intestine was opened and fixed in formalin.

\section{RESULTS}

The structure of the mucosa, as viewed under the dissecting microscope, was excellently preserved throughout the whole small intestine. Histological sections, however, showed an appearance that would be dismissed as autolysis because almost all 


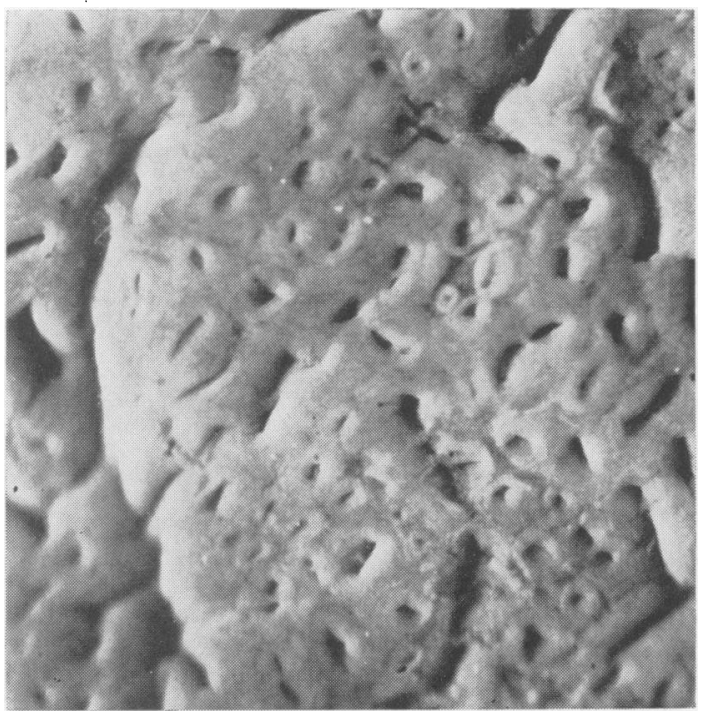

FIG. 1. Peroral jejunal biopsy taken one month before death.

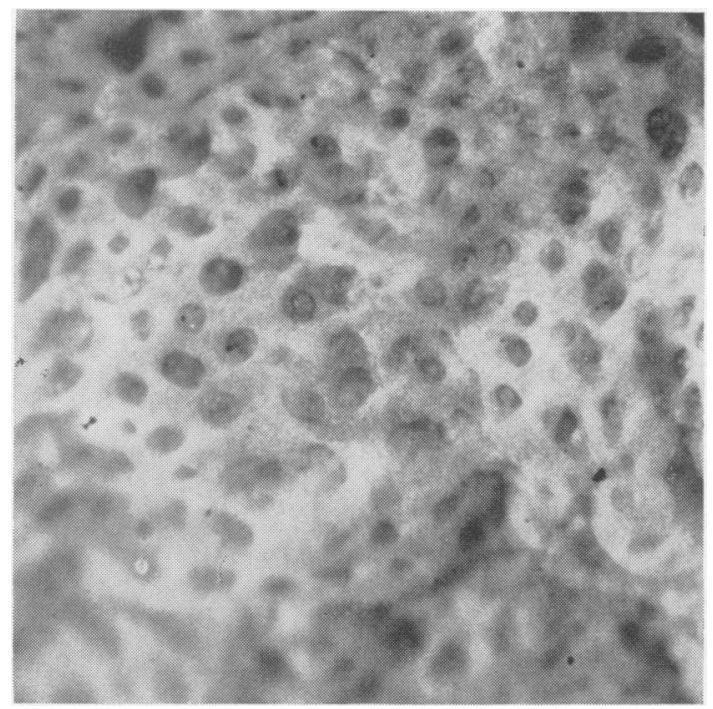

FIG. 3. Specimen no. 2: trough between mucosal folds.

the epithelial cells covering the surface were missing and those in the crypts were extremely degenerate. When the post-mortem mucosa was compared with the ante-mortem biopsies the explanation of this discrepancy was immediately apparent (Figs. 1 and 2). The visibly stained structures were the naked stroma of the epithelium; in fact the basement membrane was now the surface. Thus the orifices in the jejunum were large in the post-mortem material but like buttonholes in the biopsies. In

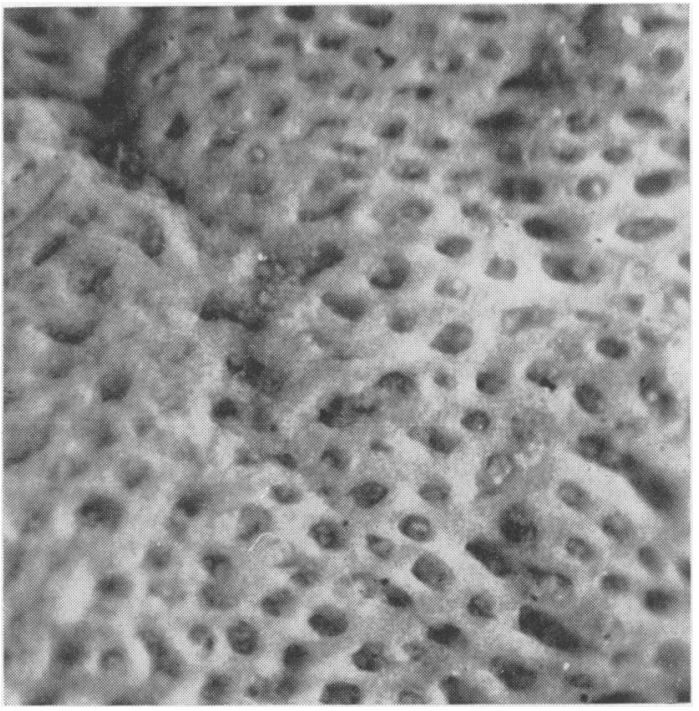

FIG. 2. Specimen no. 2: crest of mucosal fold.

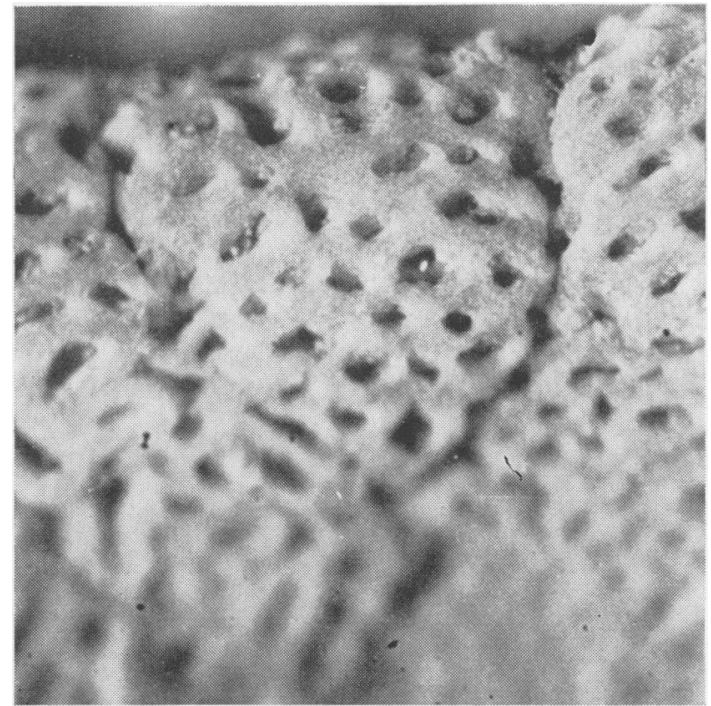

FIG. 4. Specimen no. 5: crest of mucosal fold.

these post-mortem orifices cell debris could be seen. Similarly in the lower parts of the small intestine the convolutions and leaves were thinner when compared with the surgically resected specimen and were naked structures.

OVERALL PICTURE The specimens showed a complete gradation from a flattening in the duodenum and upper jejunum to a nearly normal appearance in the terminal ileum (Figs. 2-16). With the exception 


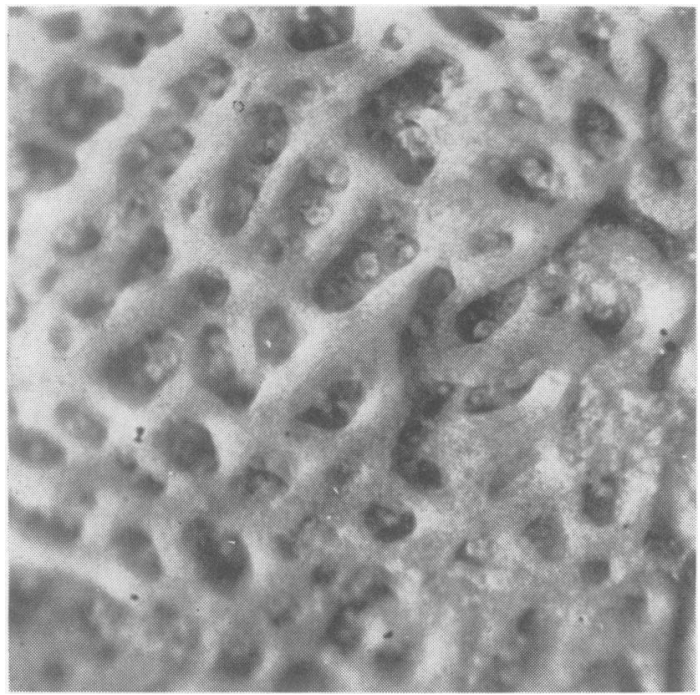

FIG. 5. Specimen no. 5: trough between mucosal folds.

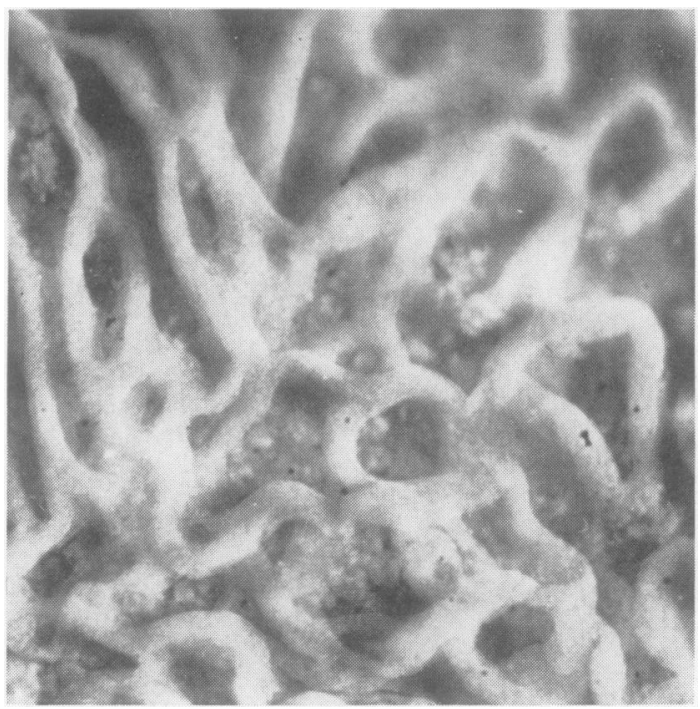

FIG. 7. Specimen no. 8: trough between mucosal folds.

of differences due to site on the mucosal folds there was little irregularity in the gradual change from top to bottom of the small intestine. As the mucosal structure became more complicated in the ileum the range of variations seen at any one level became greater.

MUCOSAL FOLDS In every specimen there was a difference between the structure on the crests of the mucosal folds compared with the troughs in between,

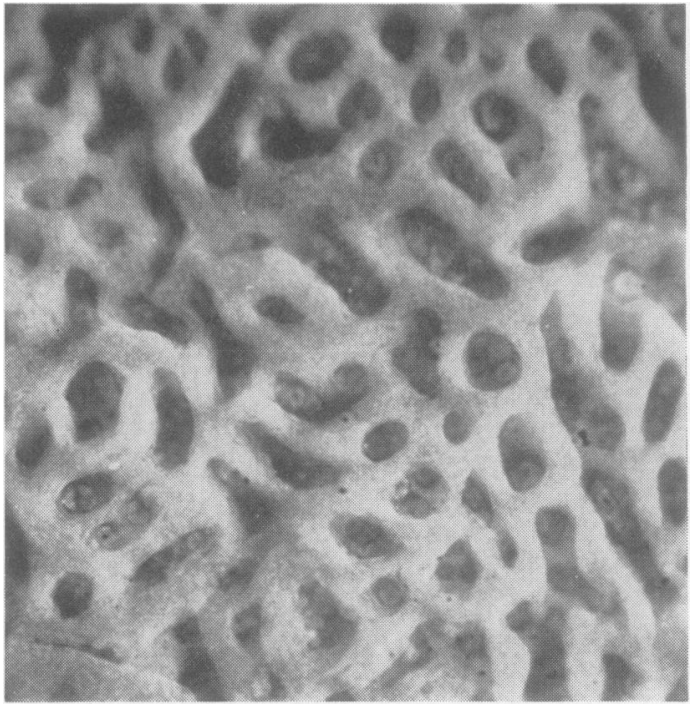

FIG. 6. Specimen no. 8: crest of mucosal fold.

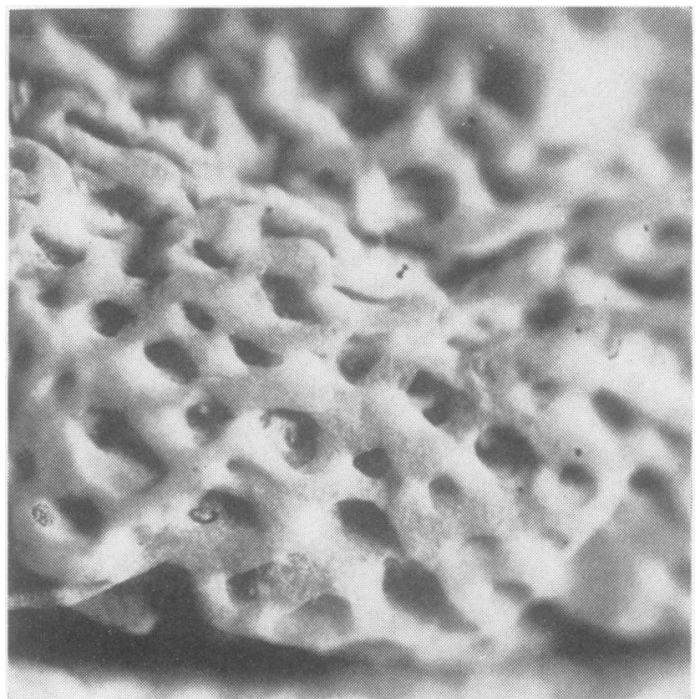

FIG. 8. Specimen no. 10: crest of mucosal fold.

but this was more marked in the mid and lower intestine than in the upper. On the crests the structures were always flatter and simpler than those in the troughs (Figs. 2-12). Where ridges and leaves were present these were orientated so they ran transversely across the folds, that is, along the luminal axis of the intestine (Fig. 11). Finger-shaped villi were not seen on the crests of the folds except in the terminal ileum, where the folds were scanty and small. 


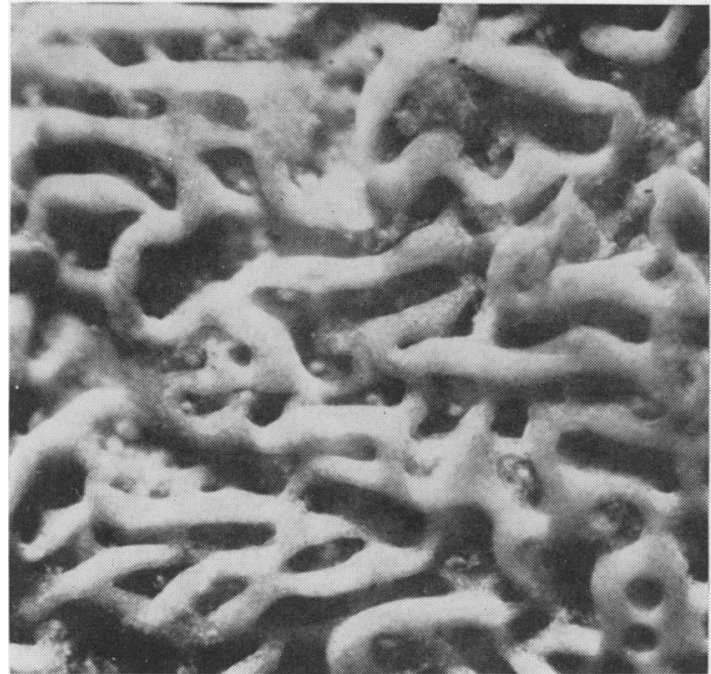

FIG. 9. Specimen no. 10: trough between mucosal folds.

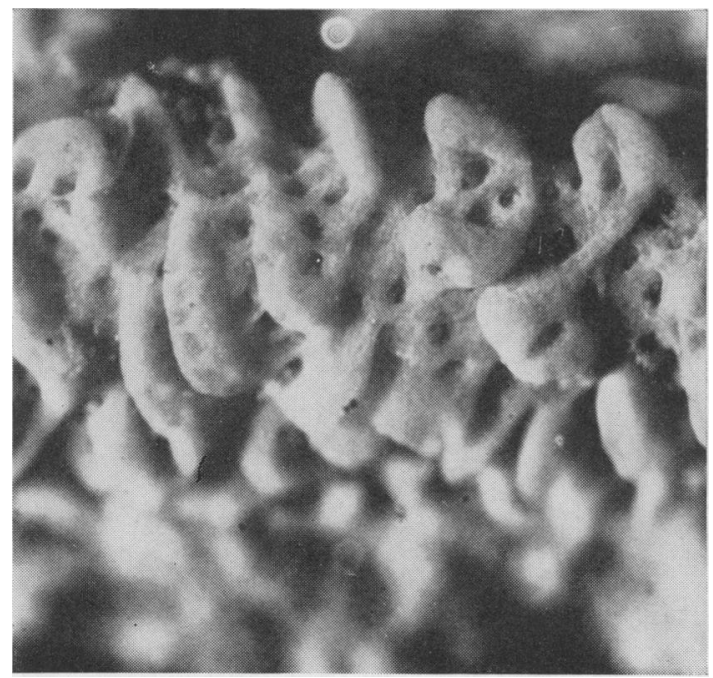

FIG. 11. Specimen no. 18: crest of mucosal fold.

TRANSITION FROM NORMAL TO FLAT APPEARANCE In the lower ileum there were easily recognizable finger-shaped villi (Fig. 16). There was, however, often a profusion of appearances with finger villi, leaves, and doughnut-like structures with a crypt opening in the centre. In between the villi of whatever shape crypt orifices could be seen. A little higher leaf-shaped structures predominated but there were also some interconnecting convolutions (Fig. 15). In the upper ileum there was an obviously

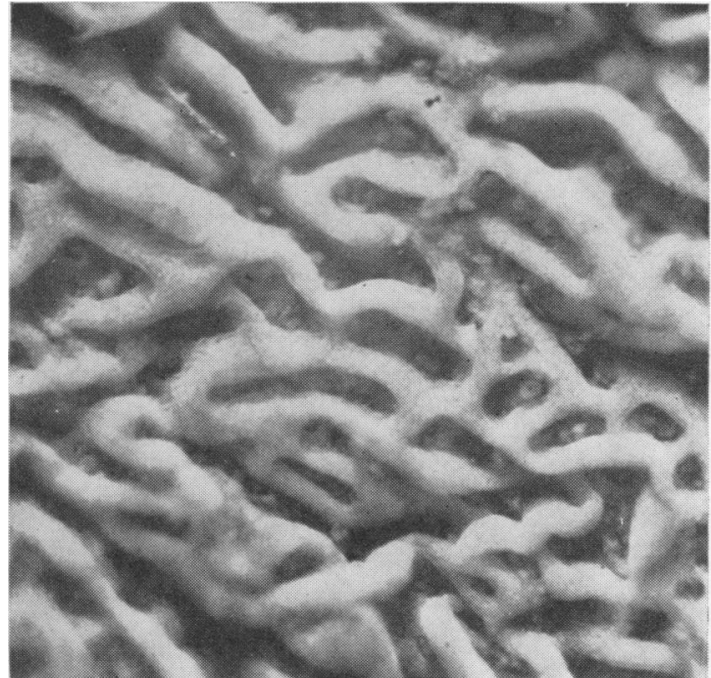

FIG. 10. Specimen no. 13: trough between mucosal folds.

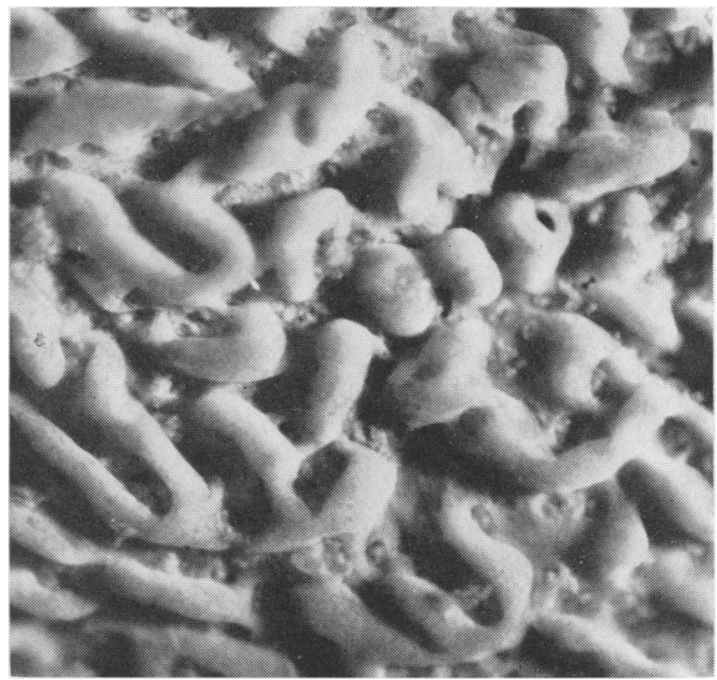

FIG. 12. Specimen no. 18: trough between mucosal folds.

convoluted pattern with a maze of ridges joining and dividing with the openings of crypts clearly visible between them and occasionally opening in the middle of a ridge (Fig. 10). At slightly higher levels the ridges became larger and were usually orientated so that most ran in one direction with slender connecting cross ridges. Higher in the jejunum the ridges became lower structures and holes between them more circular; these orifices were much larger than the crypt openings in the ileum (Fig. 6). In the 


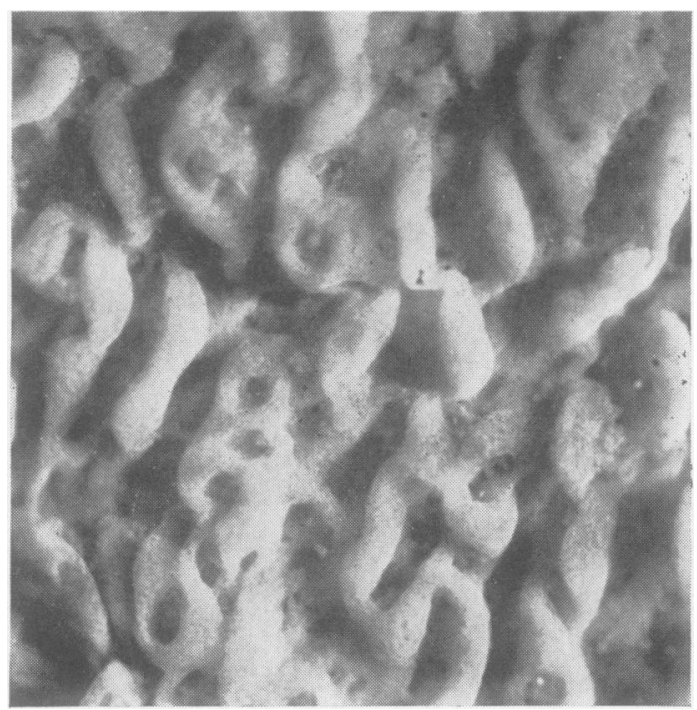

FIG. 13. Specimen no. 20: trough between mucosal folds.

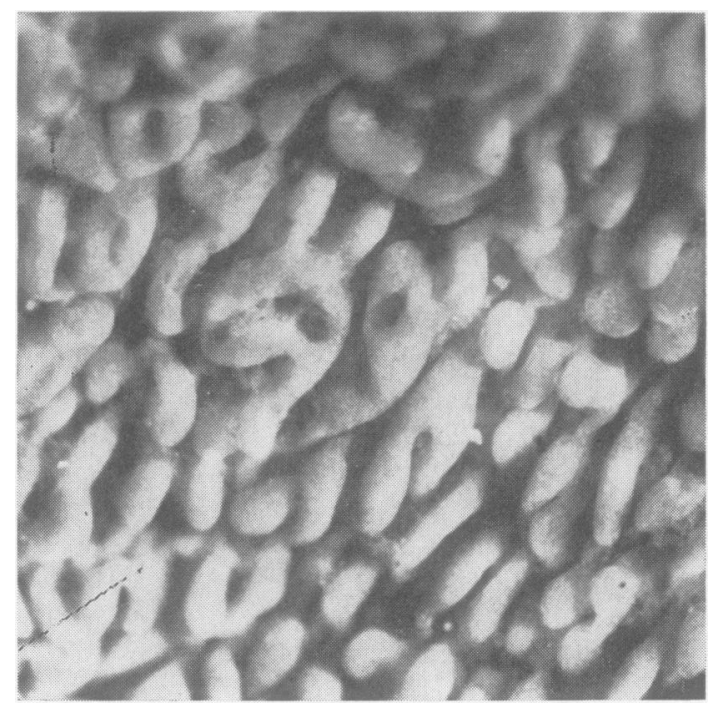

FIG. 15. Specimen no. 22: trough between mucosa folds.

upper jejunum and duodenum the mucosa on the crests of the mucosal folds, which were larger than those in the ileum, was completely flat with large orifices giving a pigskin-like appearance and at the bottom of these orifices the openings of two or three crypts could be seen (Fig. 2). In the troughs the mucosa was also flat but about the orifices the structure was slightly raised looking more like a honeycomb (Fig. 3). Over this area a crazy-paving pattern was present, more prominent on the crests

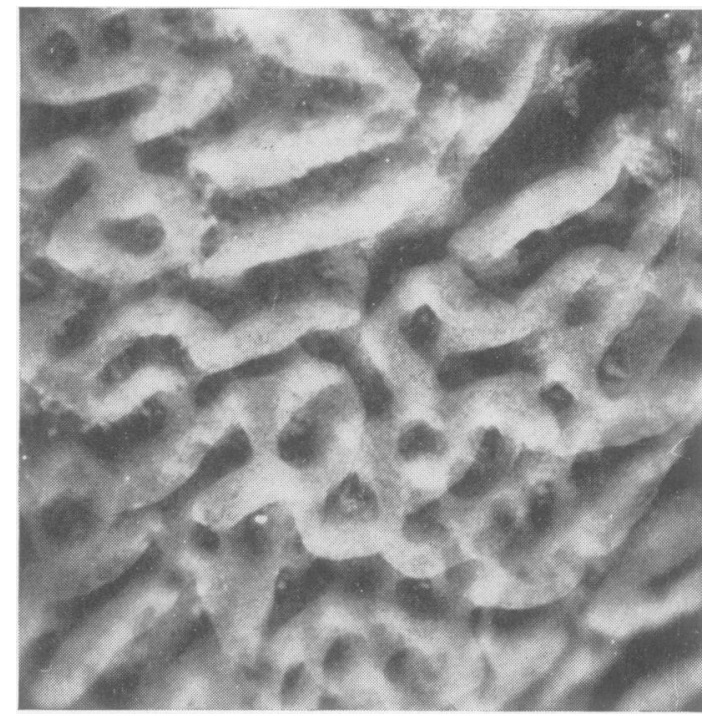

FIG. 14. Specimen no. 21: trough between mucosal folds.

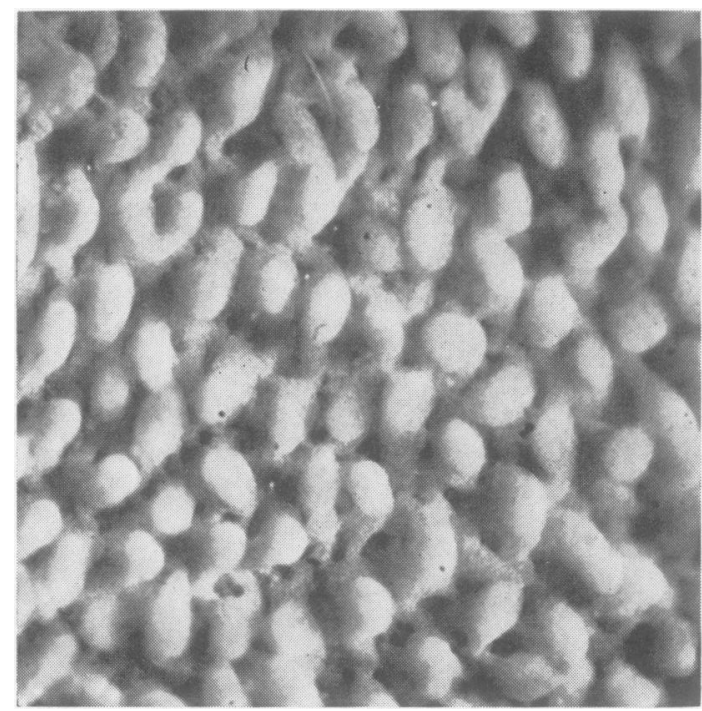

FIG. 16. Specimen no. 23: trough between mucosal folds.

of the folds (Fig. 17). This pattern delineated areas containing from five to 50 orifices.

CUT SURFACE OF MUCOSA When the specimens were cut vertically the mucosa could be examined as in a histological section. The detail here was not as good as on surface inspection but the relation of orifices to crypts could be seen. In the duodenum and upper jejunum there were more crypts than orifices and crypts appeared to join to form an orifice. In the 


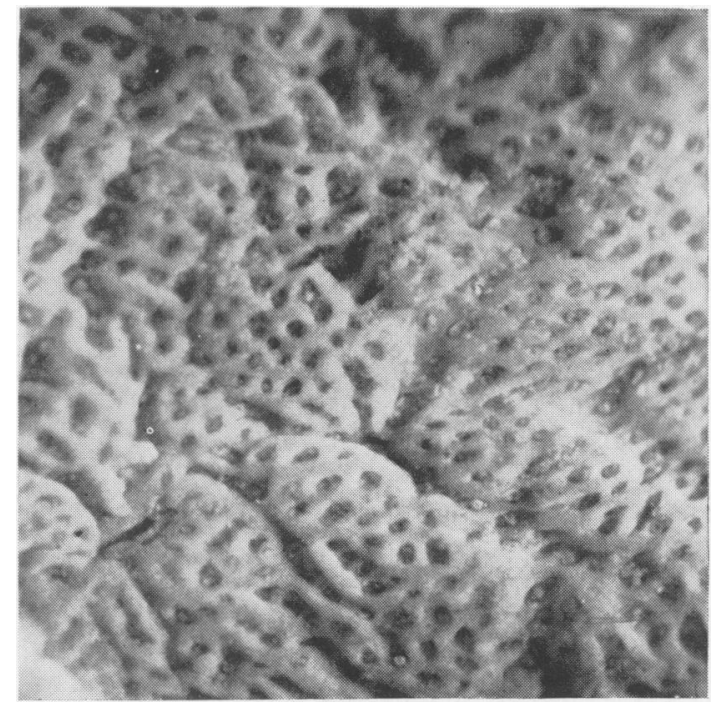

FIG. 17. Specimen no. 5: low-power view showing crazy paving pattern.

lower parts of the small intestine the crypts could be seen to open directly onto the surface. The outline of the crazy paving appeared to be gutters of the same depth as the orifices and were thus orifices extended in one direction.

\section{DISCUSSION}

In this case the whole small intestine obtained at a routine necropsy 24 hours after death was entirely satisfactory for examination by the dissecting microscope although histology showed marked autolysis. The lack of enzymes in the abnormal mucosa may have favoured the preservation but we have so far found that many other cases can be similarly examined at necropsy (Loehry and Creamer, 1965). The structure differs from biopsy and surgical specimens in that it is denuded of surface epithelium and it is the stroma which is being examined. This, however, gives a vivid view of the mucosal architecture. Although the patient was treated with chlorambucil in life it seems unlikely to have caused much change in the mucosa, as biopsies before death showed little change.

From this study certain points emerge. The mucosal abnormality is most marked in the duodenum and upper jejunum and gradually changes to a near normal appearance in the lower ileum. This is in accord with the studies where the ileum has been biopsied (Rubin et al., 1960). The gradual change from a flat to normal appearance passes through a variety of structures with a gradually increasing surface area. This can be better appreciated from the illustrations than from any written description. It is clear that a flat mucosa must be a very stable structure while convolutions, leaves, and finger villi represent a change to increasingly vulnerable and unstable projections. This is emphasized by the marked difference between the mucosa on the crests of folds and that in the more sheltered troughs. This would seem to be due to trauma and any theory of the genesis of the abnormal mucosa must take this into account.

The findings also show that abnormal mucosal structures are moulded by certain factors, presumably mainly by the intestinal movements and flow of contents. Thus the ridges on the crests of the folds always ran across the fold and not along it. Also the crazy paving pattern seen in the upper small intestine is a guttering dividing up the mucosa into small areas and would seem to be again the result of intestinal movement, allowing for buckling and stretching.

This method of examining the small intestine gives a vivid picture of the relation of crypts to villous structures. In the upper small intestine the orifices opening onto the surface appear to be common channels for more than one crypt while in the ileum the crypts open directly at the base of villi.

\section{SUMMARY}

The whole small intestine of a patient who died from a secondary coeliac syndrome due to reticulum cell sarcoma has been examined by the dissecting microscope. This report illustrates the spectrum of change from the flat upper intestinal mucosa to the almost normal lower ileum. The mucosal structures are naked of epithelial cells and represent the stromal architecture.

\section{REFERENCES}

Hindle, W., and Creamer, B. (1965). The significance of a flat small intestinal mucosa. Brit. med. $J ., 2,455-458$.

Holmes, R., Hourihane, D. O'B., and Booth, C. C. (1961). The mucosa of the small intestine. Postgrad. med.J., 37, 717-724.

Loehry, C. A., and Creamer, B. (1965). Unpublished observations.

Rubin, C. E., Brandborg, L. L., Phelps, P. C., and Taylor, H. C. Jr. (1960). Studies of celiac disease. I. The apparent identical and specific nature of the duodenal and proximal jejunal lesion in celiac disease and idiopathic sprue. Gastroenterology, 38, 28-49. 